\title{
Acidic sites in beta zeolites in dependence of the preparation methods
}

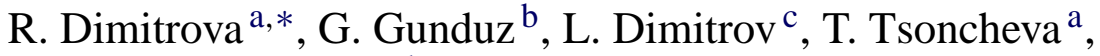 \\ S. Yialmaz ${ }^{\mathrm{d}}$, E.A. Urquieta-Gonzalez ${ }^{\mathrm{e}}$ \\ ${ }^{a}$ Institute of Organic Chemistry, Bulgarian Academy of Sciences, Acad. G. Bonchev Street bl.9, 1113 Sofia, Bulgaria \\ ${ }^{\mathrm{b}}$ Department of Chemical Engineering, Ege University, Bornova, Izmir, Turkey \\ ${ }^{\mathrm{c}}$ Institute of Catalysis, Bulgarian Academy of Sciences, 1113 Sofia, Bulgaria \\ ${ }^{\mathrm{d}}$ Department of Chemical Engineering, Izmir Institute of Technology, Izmir, Turkey \\ e Centro de Ciencias Exatas e de Tecnologia, Universidade Federal de Sao Carlos, Federal de Sao Carlos, Brazil
}

Received 27 August 2003; received in revised form 15 December 2003; accepted 21 December 2003

\begin{abstract}
Beta zeolites synthesized by different methods are compared in their structure peculiarities and acidic properties by nitrogen physisorption, IR-KBr, pyridine sorption-desorption and thermoprogrammed desorption of ammonia. It is found that mesopores could be obtained in beta zeolites with the change of the activation procedure. Methanol conversion to dimethyl ether and hydrocarbons is used as a test reaction for understanding of the acid center function.
\end{abstract}

(c) 2004 Elsevier B.V. All rights reserved.

Keywords: Acid beta zeolites; Mesostructured beta seeds; Methanol conversion

\section{Introduction}

The application of zeolites as catalysts is based upon the transformations of hydrocarbons (HC) within their framework cavities. Two important factors are well known to contribute to the activity of the zeolite catalysts-their aperture and acidity. For a given zeolite structure, it is generally observed that optimum in catalytic activity is reached at a high aluminium content, since a strong correlation has been established between the latter and the zeolite acidity [1-3]. Regarding beta zeolite, this statement is not entirely perfect, because of its peculiar pore system which is disordered and is build from channels instead of cages [4,5]. Dealumination of beta zeolite is known to occur very easily, resulting in an increase in the number of active sites. Catalytic studies demonstrate an existence of a specific combination of Brønsted, Lewis acid sites and framework defects that are affected by the procedures used for beta zeolite synthesis and temperature activation [6,7]. In the presented work, we compare the influence of the procedure of beta zeolite synthesis and its transformation into hydrogen form on

\footnotetext{
* Corresponding author. Tel.: +359-2-9609111; fax: +359-2-8700225

E-mail address: zeolab@orgchm.bas.bg (R. Dimitrova).
}

samples physical and chemical properties by several methods, including a test reaction of methanol conversion [8].

\section{Experimental}

\subsection{Materials}

Beta zeolites with modules $\left(\mathrm{SiO}_{2} / \mathrm{Al}_{2} \mathrm{O}_{3}\right)$ in a narrow range and specific surface area (BET) of $400-750 \mathrm{~m}^{2} / \mathrm{g}$ were synthesized by different methods (Table 1).

Method 1: Sample denoted as Beta(55), with $\mathrm{SiO}_{2} / \mathrm{Al}_{2} \mathrm{O}_{3}$ $=55$, was synthesized by hydrothermal crystallization of the appropriate gel as described in [9]. It was washed with deionized water and dehydrated at $373 \mathrm{~K}$ for $2 \mathrm{~h}$. Next, the sample was treated at $523 \mathrm{~K}$ for $2 \mathrm{~h}$ and finally heated from 523 to $823 \mathrm{~K}$ in an electrical furnace to burn the template off, slowly increasing the temperature. Further on, $1 \mathrm{~g}$ of it was exchanged in a $0.1 \mathrm{M} \mathrm{NH}_{4} \mathrm{Cl}$ solution $(100 \mathrm{ml})$ at $363 \mathrm{~K}$, dried and calcined to prepare the hydrogen form.

Method 2: Sample denoted as Beta(66) with $\mathrm{SiO}_{2} / \mathrm{Al}_{2} \mathrm{O}_{3}$ $=66$ was synthesized according to the steam-assisted crystallization method as described in [10]. It was converted to hydrogen form by ion exchange in a $0.1 \mathrm{M} \mathrm{NH}_{4} \mathrm{Cl}$ solution 
Table 1

Samples characteristics based on sorptive analyses

\begin{tabular}{lccccc}
\hline & $\operatorname{Beta}(20)$ & $\operatorname{Beta}(55)^{\mathrm{a}}$ & $\operatorname{Beta}(66)$ & $\begin{array}{l}m \text { Beta } \\
(66-48)^{\mathrm{a}}\end{array}$ & $\begin{array}{l}m \text { Beta } \\
(66-113)^{\mathrm{a}}\end{array}$ \\
\hline $\begin{array}{l}\text { BET }\left(\mathrm{m}^{2} / \mathrm{g}\right) \\
\begin{array}{c}\text { Dessorbed NH } \\
\quad \text { (mg eq.) }\end{array}\end{array}$ & 100 & 612 & 588 & 758 & 676 \\
$\begin{array}{l}\text { Sites relative } \\
\text { acid strength } \\
(\mathrm{mmol} / \mathrm{g})\end{array}$ & 1.00 & 0.86 & 0.56 & 0.46 & 0.53 \\
FTIR $^{\mathrm{b}}, \mathrm{L} / B$ & 0.4 & 1.15 & 1.35 & 0.016 & 0.17 \\
\hline
\end{tabular}

${ }^{a}$ Calcined at $823 \mathrm{~K}$ in electric furnace, before transformation in hydrogen form.

${ }^{\mathrm{b}} L$ is the integral intensity of the band at $1455 \mathrm{~cm}^{-1}$ in the IR spectrum of adsorbed pyridine after evacuation at $573 \mathrm{~K} ; B$ is the integral intensity of the band at $1546 \mathrm{~cm}^{-1}$ in the IR spectrum of adsorbed pyridine after evacuation at $573 \mathrm{~K}$.

at $363 \mathrm{~K}$, dried and calcined at $823 \mathrm{~K}$ for $4 \mathrm{~h}$ in an electrical furnace.

Method 3: Samples denoted as $m$ Beta(66-48) and $m$ Beta(66-113), with $\mathrm{SiO}_{2} / \mathrm{Al}_{2} \mathrm{O}_{3}=66$ (the second number in the brackets stands for the time of mesostructuring), were assembled from beta zeolite seeds [11]. The beta zeolite seeds were ripened at $373 \mathrm{~K}$ for $24 \mathrm{~h}$ from a solution with a composition: $6.7 \mathrm{~mol}$ TEAOH, $\mathrm{NaAlO}_{2}, 33.3 \mathrm{~mol}$ $\mathrm{SiO}_{2}$, and $1270 \mathrm{~mol} \mathrm{H}_{2} \mathrm{O}$, where TEAOH is tetraethylammonium hydroxide (Merck, 20\% solution in water). Further on, mesostructuring of the beta seeds was carried out in hydrothermal conditions at $323 \mathrm{~K}$ in presence of a template (cethyltetramethylammonium bromide $(0.4(\mathrm{~mol} / \mathrm{mol})$ of $\left.\mathrm{SiO}_{2}\right)$ ). Samples were taken after 24, 48, 96 and $113 \mathrm{~h}$. The samples were washed with deionized water, then ion exchanged with a $0.1 \mathrm{M} \mathrm{NH}_{4} \mathrm{Cl}$ solution, washed and dried. Further on, the occluded organic templates were removed by calcination in an electrical furnace at $823 \mathrm{~K}$ for $6 \mathrm{~h}$. The ion-exchange procedure was repeated and the samples were finally transformed in their hydrogen forms by calcination at $823 \mathrm{~K}$ for $4 \mathrm{~h}$.

For comparison, a sample denoted as $\mathrm{Beta}(20), \mathrm{SiO}_{2} /$ $\mathrm{Al}_{2} \mathrm{O}_{3}=20$, synthesized by hydrothermal crystallization and calcined up to $823 \mathrm{~K}$ under a dry airflow of $80 \mathrm{ml} / \mathrm{min}$ was used.

\subsection{Characterization procedures}

Nitrogen physisorption measurements (for micro- and mesopores analysis) were performed at $77 \mathrm{~K}$ using a static volumetric apparatus (Coulte Omnisorb 100cx) up to $p / p_{0} \sim 0.95$ as described in [12]. The specific total surface areas were calculated using Langmuir equation. Mid-IR patterns were collected on a Brucker spectrometer (Vector 22), using $\mathrm{KBr}$ pellet technique. Pyridine sorption was carried out to identify the samples acid centers. For the purpose, pyridine was sorbed on dried samples for 1 day at $423 \mathrm{~K}$ under a pyridine pressure of a few Torrs. After that, the excess amount of pyridine was vaporized in vacuum and pyridine desorption was carried out at different temperatures (423, 573 and $723 \mathrm{~K}$ ). Wafers were prepared by using $\mathrm{KBr}$ pressed disk technique $(8 \mathrm{mg}$ catalyst $/ 100 \mathrm{mg} \mathrm{KBr})$ for the IR spectra. Temperature programmed desorption of ammonia (TPDA) was performed in a conventional flow reactor supplied with a thermal conductive detector. The precise procedure had been described in [13].

\subsection{Catalytic test}

The catalytic experiments were performed in a fixed bed reactor at atmospheric pressure. The catalyst $(0.1 \mathrm{~g})$ with particle size of $0.2-0.8 \mathrm{~nm}$ was diluted with a three-fold amount of glass. The methanol conversion was studied at temperatures above $550 \mathrm{~K}$ with a WHSV of $1.5 \mathrm{~h}^{-1}$ and methanol partial pressure of $1.57 \mathrm{kPa}$, argon being used as a carrier gas. The on-line gas chromatographic analysis was done on $2 \mathrm{~m}$ Porapak $\mathrm{Q}$ and $2 \mathrm{~m}$ Durapak columns. The products yields were calculated using an absolute carbon base calibration.

\section{Results and discussion}

Upon substitution of different amount of $\mathrm{Si}$ by $\mathrm{Al}$ in the framework of beta zeolite, a local deformation of the $\mathrm{Si}-\mathrm{O}-\mathrm{T}$ bridges, by rearrangement of the oxygen atoms directly coordinated to the T-atoms occurs. The effect is well distinguished in the IR-KBr spectra by the changes of bands at around $920 \mathrm{~cm}^{-1} / 940 \mathrm{~cm}^{-1}$ (Fig. 1). According to Scholle and Veeman [14], the peak at about $940 \mathrm{~cm}^{-1}$ is pointed for silica, silica gels and various siliceous zeolites and has been assigned to the stretching vibration of $\mathrm{Si}-\mathrm{O}$ vibrators, belonging to uncoupled $\mathrm{SiO}$ tetrahedra with a hydroxyl group. Thus, the observed bands at around $920 \mathrm{~cm}^{-1} / 940 \mathrm{~cm}^{-1}$ could be assigned to framework irregularity associated with the presence of silanol nests and differently bonded extraframework aluminium species (Fig. 1).

It is noted that the band is less intensive in the spectra of Beta(55) and Beta(66) samples (Fig. 1, curves (B) and

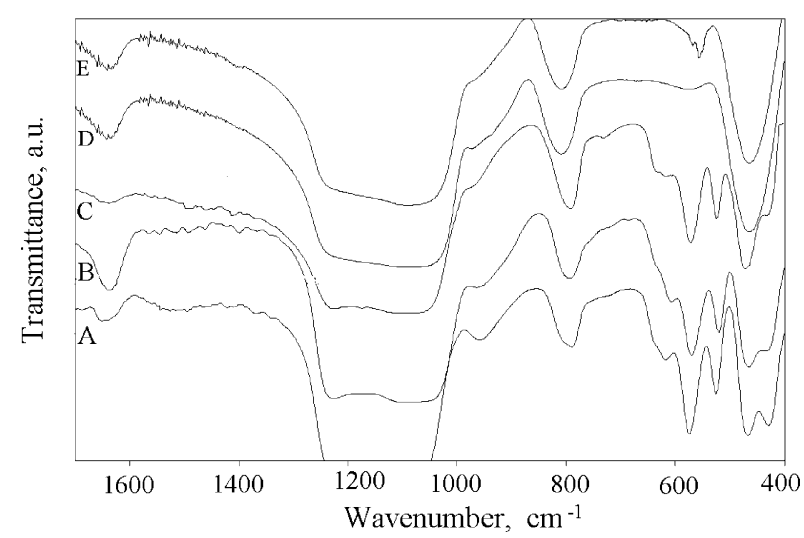

Fig. 1. IR-KBr spectra of (A) Beta(20); (B) Beta(55); (C) Beta(66); (D) $m \operatorname{Beta}(66-48)$ and (E) $m \operatorname{Beta}(66-113)$. 


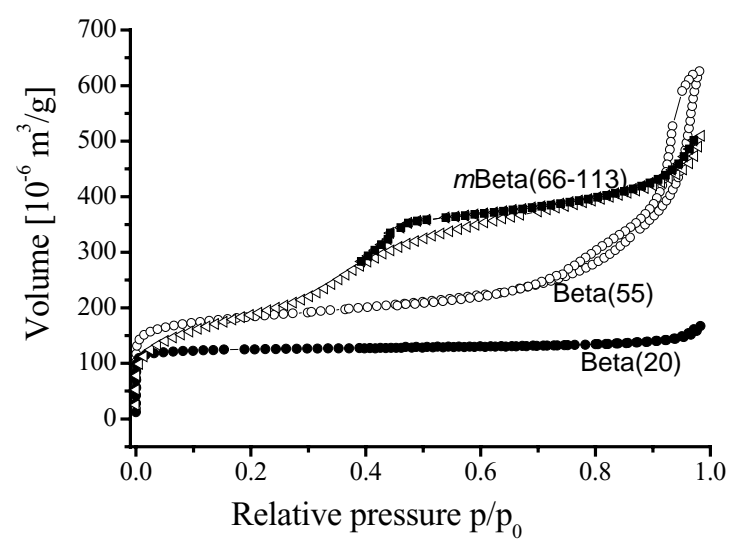

Fig. 2. $\mathrm{N}_{2}$ physisorption isotherms $(77 \mathrm{~K})$ for $(\bigcirc) \operatorname{Beta}(55)$ and $(\boldsymbol{\Delta}, \triangleleft)$ $m \operatorname{Beta}(113)$ in comparison to $(\mathbf{O}) \operatorname{Beta}(20)$.

(C)) in comparison with sample (Beta(20)). So, differences in beta framework distortions depending on the methods of synthesis could be assumed [15]. A decrease of the band at $940 \mathrm{~cm}^{-1}$ is also observed for samples obtained by method 3 (Fig. 1, curves (E) and (D)). In these curves a change in the band at about $570 \mathrm{~cm}^{-1}$ is well distinguished too. As established in [16] both bands are typical for $(\mathrm{O} 3) \mathrm{Si}-\mathrm{O}^{-}$ groups and associated with the presence of non-bridging oxygen in gels, and amorphous silica. For $m$ Beta(66-113) sample, the band at $570 \mathrm{~cm}^{-1}$ splits $\left(561\right.$ and $\left.620 \mathrm{~cm}^{-1}\right)$, and the latters are more intensive and narrowed in comparison with $m$ Beta(66-24). So, it is probable the development of crystalline beta phase with the time of mesostructuring.

Certain differences are observed in sample nitrogen adsorption/desorption isotherms. BETA(20) sample has an adsorption isotherm of type I (typical for microporous adsorbents, like zeolites) (Fig. 2). In contrast, the adsorption isotherms of BETA(55) and $\operatorname{BETA(66)~samples~resemble~}$ the one of the micro-meso porous type material (Fig. 2-not shown for Beta(66)). Their capillary condensation loops are observed at high partial pressure and the measured specific surface area of meso and macropores (about $220-230 \mathrm{~m}^{2} / \mathrm{g}$ ) is nearly five times bigger than the one of Beta(20) sample $\left(43 \mathrm{~m}^{2} / \mathrm{g}\right)$.

An increase in the average half width of the pores is also registered (about $0.68 \mathrm{~nm}$ for samples synthesized by methods 1 and 2 in relation to $0.50 \mathrm{~nm}$ for Beta(20)). So, beta samples synthesis with bimodal pore systems is suggested by applying methods 1 and 2 . The adsorption isotherms of $m$ Beta(66-48) and $m \operatorname{Beta}(66-113)$ samples are of type IV with loops of capillary condensation of mixed type B and E (Fig. 2). The capillary condensation loop of type $\mathrm{E}$ is observed in the range of $p / p_{0}=0.4-0.5$ and could be assigned to framework mesoporosity. The capillary condensation loop of type B appears near to complete saturation and could be assigned to interparticle mesoporosity. The specific surface area of meso and macropores increases about four times in comparison with $\operatorname{BETA}(55)$ and $\operatorname{BETA}(66)$ samples and reaches 985 and $768 \mathrm{~m}^{2} / \mathrm{g}$ for $m \operatorname{Beta}(66-48)$ and

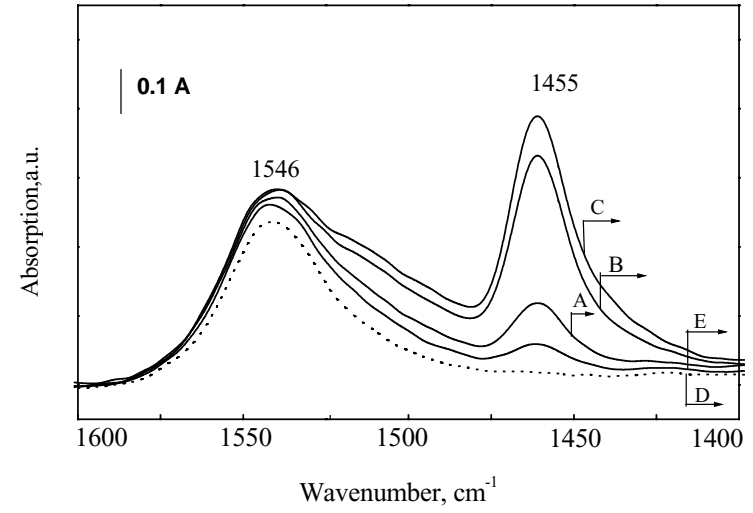

Fig. 3. IR spectra of pyridine species after adsorption at $423 \mathrm{~K}$ ( $p=2$ Torr) followed by evacuation at $523 \mathrm{~K}$ : (A) $\operatorname{Beta}(20)$; (B) Beta(55); (C) Beta(66); (D) $m \operatorname{Beta}(66-48)$ and (E) $m \operatorname{Beta}(66-113)$.

$m$ Beta(66-113), respectively. An increase in the average half with of the pores is also achieved, as it is almost the same (about $1.064 \mathrm{~nm}$ ) for both $m$ Beta samples. Hence, the samples synthesized by the method 3 could be characterized as typical mesoporous beta materials.

Samples total acidity, determined by TPDA, are presented in Table 1. Close values of desorbed ammonia (1.09-1.34 mg/eq.) are found for all samples with the exception of Beta(55). However, the calculated value for sites relative acid strength, based on its silica module and the amount desorbed ammonia, significantly differs.

In order to explain this fact, the nature of the acid sites has been studied by pyridine sorption, assuming that all aluminium is detected (Fig. 3). The concentration of tetrahedral aluminium is known to be proportional to the concentration of the Brønsted acid sites, whose interaction with pyridine yields pyridinium cations with characteristic IR bands at ca. $1546 \mathrm{~cm}^{-1}$. The integral intensity of the peak observed at ca. $1455 \mathrm{~cm}^{-1}$ is used for estimating the concentration of Lewis acid site $[17,18]$. The detected integral intensity of the IR band at ca. $1546 \mathrm{~cm}^{-1}$ is close for all samples, while the intensity of the band at ca. $1455 \mathrm{~cm}^{-1}$ significantly decreases in the order $\operatorname{Beta}(66)>\operatorname{Beta}(55)>\operatorname{Beta}(20)>m \operatorname{Beta}(66-113)$ $>m$ Beta(66-48). In Table 1, the ratio between the Lewis and Brønsted sites elucidated by the IR spectra is given. It is found that Beta(55) and Beta(66) samples display almost an order of magnitude higher amount of Lewis centers in comparison with both $m$ Beta samples. It must be mentioned, however, that all samples with $\mathrm{SiO}_{2} / \mathrm{Al}_{2} \mathrm{O}_{3}=66$, but synthesized by different methods, have almost equal value for sites relative acid strength, while the number of Lewis sites differs essentially (Table 1). So, the presence of Brønsted sites with different strength could be assumed for the bimodal pore sample in comparison with the mesoporous materials. This conclusion is also confirmed regarding the reference (Beta (20)) sample, where stronger acidity at lower number of Lewis sites is found.

The understanding of the acid center behavior has been accomplished by comparative catalytic studies. Methanol 


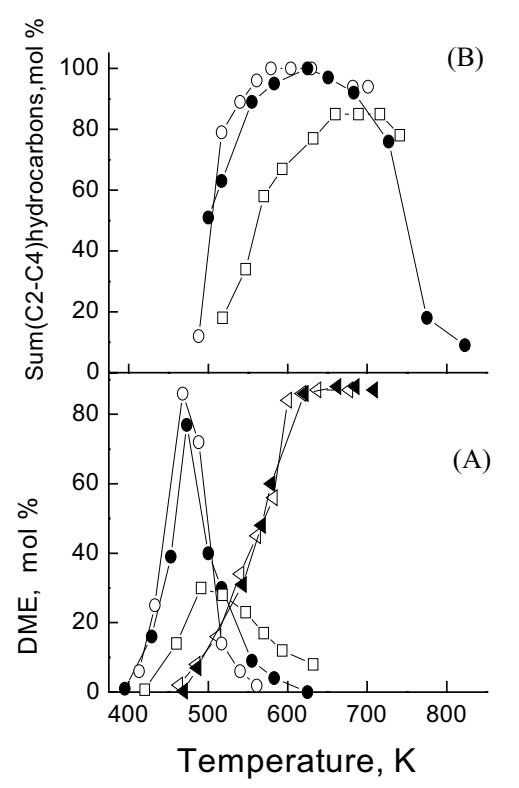

Fig. 4. DME (A) and hydrocarbons (B) yields vs. temperature on (О) $\operatorname{Beta}(20) ;(\bigcirc) \operatorname{Beta}(55) ;(\square) \operatorname{Beta}(66) ;(\triangleleft) m \operatorname{Beta}(66-48)$ and (४) $m \operatorname{Beta}(66-113)$.

conversion to dimethyl ether (DME) and hydrocarbons has been used as a test reaction for characterization of the total and Brønsted acidity (Fig. 4) [19,20]. All samples show high catalytic activity to DME, as the conversion on both $m$ Beta catalysts is detected at relatively higher temperatures (Fig. 4A). Besides, negligible amount of $\left(\mathrm{C}_{2}-\mathrm{C}_{4}\right)$ hydrocarbons is determined below $700 \mathrm{~K}$ on them too. Thus, the obtained catalytic results and the found low number of Lewis sites, evidence for the presence of week Brønsted sites in the materials with mesoporous structure.

At the same time, the higher activity of $\operatorname{Beta}(20)$ for the formation of $\left(\mathrm{C}_{2}-\mathrm{C}_{4}\right)$ hydrocarbons (Fig. 3B) and the determined low number of Lewis acid sites in it verify the existence of strong Brønsted sites. Therefore, the synthesis of beta samples with micro, micro-meso, or mesoporous property influences not only the nature of the acid sites (Brønsted or Lewis), but their strength as well. Probably, the latter strongly depends on the acid sites localization and environment in the different micro- or mesoporous structure.

\section{Conclusions}

Using different preparation methods, beta structures with micro, micro-meso and mesoporous properties are obtained. The nature and strength of the formed acidic sites depends on their localization in the micro- and mesopores.

\section{References}

[1] A. Corma, H. García, Catal. Today 38 (1997) 257.

[2] F. Vaundr, F. Di Renzo, F. Fajula, F. Schulz, J. Chem. Soc., Faraday Trans. 94 (1998) 617.

[3] J. Sauer, J. Mol. Catal. 54 (1989) 312.

[4] J.B. Higgins, R.B. Lapierre, J.L. Schlenker, A.C. Rohrman, J.D. Wood, G.T. Kerr, W.J. Rohbaugh, Zeolites 8 (1988) 455.

[5] H. van Koningsveld, Zeolites 12 (1992) 114.

[6] I. Kiricsi, C. Flego, G. Pazzuconi, J. Phys. Chem. 98 (1994) 4624.

[7] M. Muller, G. Harvey, R. Prins, Micropor. Mesopor. Mater. 34 (2000) 135.

[8] T. Tsoncheva, R. Dimitrova, in: Proceedings of the 9th International Symposium on Heterogeneous Catalysis, Varna, Bulgaria, 2000, p. 465.

[9] U. Lohse, B. Altrichter, R. Donald, R. Fricke, K. Jansen, B. Parlitz, E. Schreier, J. Chem. Soc., Faraday Trans 92 (1996) 159.

[10] M. Matsukata, T. Osaki, M. Ogura, E. Kikuchi, Micropor. Mesopor. Mater. 56 (2002) 1.

[11] L. Liu, W. Zhang, T.J. Pinnavaia, Angew. Chem. 113 (7) (2001) 1295.

[12] N. Beşün, F. Özkan, G. Gűnűz, Appl. Catal. A: Gen. 224 (2002) 285.

[13] R. Dimitrova, Y. Neinska, M. Mihályi, T. Tsoncheva, M Spassova, React. Kinet. Catal. Lett. 74 (2001) 353.

[14] K.F. Scholle, W. Veeman, Zeolites 5 (1985) 118.

[15] C. Jia, P. Massiani, D. Barthomeuf, J. Chem. Soc., Faraday Trans. 89 (1993) 3659.

[16] M. Decottignies, J. Phalippon, J. Zarzycki, J. Mater. Sci. 13 (1978) 2605.

[17] S. Krijnen, R. Harmsen, H. Abberihuis, J.H. van Hoof, R. van Santen, Chem. Commun. (1999) 501.

[18] V. Zholobenki, L. Kustov, B. Kazanski, E. Loeffler, U. Lohse, G. Oehlmann, Zeolites 11 (1991) 132.

[19] T. Tsoncheva, R. Dimitrova, Appl. Catal. A: Gen. 225 (2002) 101.

[20] J. Jancen, E. Creyghton, S. Njo, H. van Koningsveld, H. van Bekkum, Catal. Today 38 (1997) 205. 